\title{
Attitudes and stigma towards seeking professional psychological help among college going students
}

\author{
Vidyadayini Shetty ${ }^{1}$, Tanya Jain ${ }^{2}$ \\ ${ }^{1}$ Associate Professor, Department of Psychology, NK College, Malad, Mumbai. \\ ${ }^{2}$ Student Counselor, NK College, Malad, Mumbai. \\ E-mail-vidhi33@gmail.com
}

\begin{abstract}
Background: People with psychological problems often refuse to seek help due to the stigma associated with mental illness. This often serves as a barrier when seeking professional psychological help even when the need arises. The purpose of the present study was to explore the relationship between attitudes and stigma towards seeking professional psychological help among college students with and without prior counseling experience. The study also aimed to explore the gender differences in attitudes and stigma toward seeking professional psychological help among college students with and without prior counseling experience.

Methods: 71 students with no prior counseling and 61 students that received prior counseling were administered the Attitudes toward seeking professional psychological help scale (ATSPPHS) and Self stigma of seeking help scales. The data was analyzed statistically and the results were presented.

Results: Quantitative data were analyzed using t test and correlation method. The findings indicate that stigma has a significant inverse relationship with the attitudes towards seeking professional counseling among college students. The study also reveals that there is no significant gender difference in attitudes and stigma among help seekers and non seekers.

Conclusion: A large scale study is needed to identify the factors influencing the attitudes of students towards counselling. To bring about attitudinal change it is important to create awareness about the need for seeking professional help in times of personal crises. Schools and colleges must appoint qualified counsellors who can make students aware of the benefits of counselling and also about its confidentiality which can help to reduce the stigma towards counselling and counsellors.
\end{abstract}

Key words: stigma, college students, attitudes, professional psychological help, counseling

(Received $-29^{\text {th }}$ April 2016, Peer Review done $-21^{\text {st }}$ May 2016, Accepted $-10^{\text {th }}$ June 2016)

\section{INTRODUCTION}

The past few years have shown a steady increase in the number of mental health services provided in India. This has been mainly attributed to the need arising due to changing lifestyles, work pressure and the impact of western culture. In spite of the steady increase in the number of mental health service centres, yet the number of people reaching out to these services is comparatively less than in other countries. According to Carson and Chowdhury [1], Counselling or therapy is generally an unknown, misunderstood or devalued enterprise in India. They further explain that therapy or psychotherapy in particular are generally viewed in negative terms and are usually associated by Indians with hospitalised treatment of mentally or emotionally ill individuals. As a result most Indians do not have a positive approach towards counselling. Moreover Indian society is more collectivistic in nature where integrated families live together and there is more inter dependency than in western culture. According to Chadda and Deb [2], in 
collectivistic societies like ours, the self is defined relative to others, and is concerned with feelings of belongingness, dependency, empathy, and reciprocity, and is focused on small, selective in-groups at the expense of out-groups. Relationships with others are emphasized, while personal autonomy, space and privacy are considered secondary. In such an environment the attitude towards seeking psychological help can be negative especially among college students as their need of social approval is more than adults.

Attitudes are evaluations of a particular person, behaviour, belief, or concept. Attitudes influences behaviour. The strength of the link between particular attitudes and behaviour varies, but generally people strive for consistency between their attitudes and their behaviour [3]. Cross cultural studies have also shown a major difference in the attitudes of western and non-western societies in seeking professional psychological help. This is mainly because different cultures have different connotations regarding mental illness and psychological disorders. After a thorough analysis of the literature as well as a careful review of their findings, Fischer and Turner [4] identified four primary attitudinal dimensions that influenced one's attitude towards seeking professional psychological help which are (1) recognition of need for psychotherapeutic help, (2) stigma tolerance, (3) interpersonal openness, and (4) confidence in mental health practitioners.

In Indian context, too the attitude of college students remain less positive as there is stigma attached towards seeking professional help and their inability to handle their problems making them look incapable in front of their friends. There are many societies that view psychopathology as threatening and uncomfortable, and these attitudes frequently foster stigma and discrimination towards people with mental health problems. Several studies show that stigma usually arises from lack of awareness, lack of education, lack of perception, and the nature and complications of the mental illness, for example odd behaviours and violence [5].

Over the past few decades our society has undergone a lot of changes especially due to rapid urbanisation, the breakdown of joint family system, changing lifestyles, conflicting value systems have put tremendous pressure on the youth today. As a result, the need for mental health services is more pronounced today. Keeping all these views in mind the following hypotheses were formulated and the present study was conducted to assess the barriers towards seeking professional psychological help in today's youth.

\section{METHODOLOGY}

\section{Hypothesis}

1. There will be no significant difference in the attitudes and the stigma toward seeking psychological help among college students with and without prior counseling experience.

2. There will be no significant gender difference in the attitudes and the stigma toward seeking professional psychological help among college students with and without prior counseling experience.

3. There will a significant negative relationship between the stigma and the attitudes towards seeking professional psychological help among college students.

\section{Sample}

Random and purposive sampling method was used to collect the data. The sample consisted of 132 students, 65 girls and 67 boys from Degree College. Data were acquired by self-administered structured questionnaires.

\section{Tools used}

\section{Attitudes toward seeking professional psychological help scale (ATSPPHS) [6]}

The ATSPPHS is a 10 item scale which is a revision of the original 29-item ATSPPHS (Fischer \& Turner, 1970). The items are answered on a 4-point Likert scale ranging from "disagree" to "agree;" The total score ranges from 0 to 30 , with higher scores indicating more positive attitudes towards seeking help. The correlation between the longer version and the revised version was 0.87 , indicating that they are measuring the same construct. Internal consistency was .84 and test-retest reliability was 0.80

Self- Stigma of seeking help (SSOSH) [7] 
The SSOSH is a 10-item self-stigma scale which is rated on a 5-point Likert scale ranging from 1 (strongly disagree) to 5 (strongly agree). The score ranges from 10 to 50 , with higher score indicating greater selfstigma. The Cronbach's alpha reliability of the scale ranged from 0.86 to 0.90 , indicating good reliability. In addition, test-retest reliability was 0.72

A self-constructed scale was developed to explore the nature of past concerns among college students and to gather data about each participant to examine the attitudes towards seeking professional counseling.

\section{Procedure}

For prior counseling group, the participants who attended 3 to 4 counseling sessions and who could read and understand English were recruited for the study. They were informed about purpose of the study and all the three scales were administered with prior consent. Students of the Degree College without prior counseling experience were randomly selected. The scales were administered in the class room with the Professor In- charge's permission. The students completed the scales within 20 minutes.

\section{STATISTICAL ANALYSIS}

The difference on scores on various scales between males and females was assessed using the independent samples $t$ test while correlation between all variables was done using Pearson's correlation. The data was analyzed and tabulated

\section{RESULTS}

Table 1 indicates that there are no significant statistical differences in the mean scores of the ATSPPH and the SSOSH between students with prior counseling and students without counseling experience.

In the present study no significant statistical differences in the mean scores of the ATSPPH and the SSOSH between girls and boys with and without prior counseling experience were established (Table 2). The mean scores of the ATSPPHS and the SSOSH of girls and boys indicate a moderate stigma and attitude towards seeking professional psychological help.

The results of this study indicated that stigma has a significant inverse relationship with the attitudes towards seeking professional counseling among college students (Table 3). The surprising fact was that the mean scores of the stigma among young girls and boys who have prior counseling experience was not significantly lower than 'No counseling' group (Table 1).

Table 1 - Analysis of scores on the scales between various groups

\begin{tabular}{|ccccc|}
\hline Scales & $\begin{array}{c}\text { Group that has } \\
\text { undergone } \\
\text { counseling } \\
(\mathrm{n}=71)\end{array}$ & $\begin{array}{c}\text { Group with no } \\
\text { prior counseling } \\
(\mathrm{n}=61)\end{array}$ & $\mathrm{t}$ value & $\mathrm{p}$ value \\
\hline $\begin{array}{c}\text { ATSPPH } \\
\text { (Mean } \pm \text { SD) }\end{array}$ & $16.36 \pm 5.19$ & $15.93 \pm 5.05$ & 0.5 & $0.6176 \mathrm{NS}$ \\
\hline $\begin{array}{c}\text { SSOSH } \\
\text { (Mean } \pm \text { SD) }\end{array}$ & $23.0 \pm 4.83$ & $24.38 \pm 5.55$ & 1.748 & $0.0829 \mathrm{NS}$ \\
\hline
\end{tabular}

NS - not significant (Statistics done using unpaired t test) 
Table 2 - Effect of gender on various groups

\begin{tabular}{|c|c|c|c|c|c|c|c|c|c|c|}
\hline & & & No $\mathrm{co}$ & seling & & & & Prio & unselir & \\
\hline & & $\mathrm{AT}^{\prime}$ & $\mathrm{PH}$ & SSC & & & ATs & $\mathrm{PH}$ & & \\
\hline & $\mathrm{N}$ & Mean & SD & Mean & SD & $\bar{N}$ & Mean & $\mathrm{SD}$ & Mean & $\overline{\mathrm{SD}}$ \\
\hline Female & 34 & 16.76 & 4.55 & 23.65 & 5.73 & 31 & 17.00 & 5.57 & 21.84 & 5.28 \\
\hline Male & 37 & 15.16 & 5.42 & 25.05 & 5.37 & 30 & 15.70 & 4.77 & 24.20 & 4.05 \\
\hline $\mathrm{t}$ value & 71 & & & & & 61 & & & & \\
\hline $\mathrm{p}$ value & & 0.17 & NS & 0.288 & & & 0.33 & NS & 0.05 & NS \\
\hline
\end{tabular}

Table 3 - Correlation between various scales

\begin{tabular}{|ccccc|}
\hline & \multicolumn{2}{c|}{ No counseling } & \multicolumn{2}{c|}{ Prior counseling } \\
& ATSPPH & SSOSH & ATSPPH & SSOSH \\
\hline ATSPPH & 1.000 & - & 1.000 & - \\
\hline SSOSH & $-.269^{*}$ & 1.000 & $-.305^{* *}$ & 1.000 \\
\hline
\end{tabular}

${ }^{*} \mathrm{p}<0.05,{ }^{* *} \mathrm{p}<0.001$ (Pearson's correlation used)

\section{DISCUSSION}

Studies reveal that gender also has a significant impact on the attitudes toward seeking professional psychological help. However, in the present study no significant statistical differences in the mean scores of the ATSPPH and the SSOSH between girls and boys with and without prior counseling experience were established. The mean scores of the ATSPPHS and the SSOSH of girls and boys indicate a moderate stigma and attitude towards seeking professional psychological help. Though the means are not significant, the mean scores indicate that girls with and without prior counseling experience have more favorable attitude towards seeking professional help than boys. However, the mean scores of the stigma scale indicate greater stigma among girls in seeking help than boys. This may be due to the cultural differences existing in the Indian society.

Fisher \& Turner [4] proposed that one's attitude towards seeking professional psychological help directly influences one's counseling-seeking behaviors. Past studies indicate that there is a significant gender difference in attitudes toward seeking psychological help [8]. Females tend to be more willing to seek counseling and have a more positive attitude toward counseling than males. The present study was not consistent with past findings.

A vast number of studies in the area of seeking professional help to deal with psychological problems imply that a positive attitude towards seeking professional help influences help-seeking behavior. Researchers have identified some factors that inhibit individuals from seeking professional psychological help. The most cited reason is the stigma attached to mental health services which deters them from seeking professional help. The present study explored the relationship between attitudes towards seeking professional psychological help and stigma attached to help-seeking behavior. The results of this study indicated that stigma has a significant inverse relationship with the attitudes towards seeking professional counseling among college students. One contrary finding was that $91.1 \%$ girls and $81.0 \%$ boys reported that they were not fearful if other people found about them seeing a counselor. This reflects faking good behaviors observed in the socio-demographic scale that improve their social desirability.

Longitudinal studies show that college is a universally stressful period which potentially leads to an increase in psychological distress. The college environment has a number of stressors that are academic, 
social, vocational, and personal in nature. In the present times, few colleges and schools appoint qualified counselors to help students deal with their personal problems. Help-seeking literature state that the problems for which students seek counseling are numerous, including: (a) post-traumatic stress, (b) physical illness, (c) substance abuse, (d) vocational planning, and (e) academic concerns. However, studies indicate that the primary reasons for seeking professional help are educational and vocational in nature. In the present study it was found that in the prior counseling group, $41.9 \%$ girls and $36.6 \%$ boys sought help for academic reasons, $48.3 \%$ girls and $36.6 \%$ boys for personal reasons and $38.7 \%$ girls and $63.3 \%$ boys for career/vocations. The results indicate gender differences in the reasons for seeking help.

Regarding their experience with counseling, $61.2 \%$ girls and 50\% boys responded that they did not find them effective. Counselors need to devote specific attention to, case management, interventions and techniques used in counseling. Continuous professional development and training should then be provided to these counselors. Counselors need to be aware of the unique challenges faced by college students [9].

From the self constructed scale, it was found that the top presenting concerns as reported by girls included academics, family relationships, depression, irritability and anger, and self confidence issues. For boys the top presenting concerns experienced included academics, followed by self confidence issues, depression, and irritability and anger, and family relationships.

Approximately $40.9 \%$ of the participants indicated that they have sought help from family members for dealing with their past concerns. However, the majority of the participants $(49.2 \%)$ felt they were strong enough to deal with it on their own, which is of a major concern. This finding was not consistent with participants reporting that they were not fearful of others knowing about them seeking help from a counselor. The reasons for not approaching a counselor need to be studied, so that counseling services are made more accessible.

The sample size is too small to generalize the results of the current study. Only those participants who could read and understand English were selected. Participants of various age groups and backgrounds should also be included as counseling services are needed by all age groups.

A large scale study is needed to identify the factors influencing the attitudes of students towards counseling. To bring about attitudinal change it is important to create awareness about the need for seeking professional help in times of personal crises. Schools and colleges must appoint qualified counselors who can make students aware of the benefits of counseling and also about its confidentiality which can help to reduce the stigma towards counseling and counselors. Colleges should provide ecounseling programs which will help to deal with confidentiality concerns. Continuous training and development of counselors is also the need of the hour.

\section{CONCLUSIONS}

The present study indicates that there was a significantly inverse relationship between help-seeking behavior and stigma among girls and boys. The attitudes and the stigma towards seeking professional help were found to be of moderate level among college going girls and boys. There were no gender differences in attitude and stigma towards seeking professional psychological help. It was also observed that there is no difference in the attitude and the stigma among help seekers and non-help seekers.

\section{REFERENCES}

1. Carson DK, Chowdhury A. Family therapy in India: A new profession in an ancient land?. Contemp Fam Ther 2000;22(4):387-406.

2. Chadda RK, Deb KS. Indian family systems, collectivistic society and psychotherapy. Indian J Psychiatry 2013;55(Supp1 2):S299-309.

3. Feldman DB, Crandall CS. Dimensions of mental illness stigma: What about mental illness causes social rejection?. J Soc Clin Psychol 2007;26(2):137-54.

4. Fischer EH, Turner JI. Orientations to seeking professional help: development and research utility of an attitude scale. Journal of consulting and clinical psychology. 1970;35(1):79-90.

5. Arboleda-Florez JU. What causes stigma?. World Psychiatry 2002;1(1):25-8. 
6. Fischer EH, Farina A. Attitudes toward seeking professional psychologial help: A shortened form and considerations for research. J College Stud Dev 1995;36(4)368-73.

7. Vogel DL, Wade NG, Haake S. Measuring the self-stigma associated with seeking psychological help. J Couns Psychol 2006;53(3):325-37.

8. Yoshii H, Watanabe Y, Mazumder AH, Kitamura H, Akazawa K. Stigma toward Schizophrenia among Parents of High School Students. Global J Health Sci 2013;5(6):46-53.

9. Greenidge WL. Attitudes towards seeking professional counseling: The role of outcome expectations and emotional openness in English-speaking Caribbean college students in the US and the Caribbean (Doctoral dissertation, University of Central Florida Orlando, Florida).

$$
\begin{aligned}
& \text { Acknowledgements - Nil } \\
& \text { Source of Funding - Nil } \\
& \text { Conflict of Interest - Nil }
\end{aligned}
$$

